\title{
Comment on Mohammed et al.: Intramedullary tibial nailing in distal third tibial fractures: distal locking screws and fracture non-union
}

\author{
Devdatta Suhas Neogi • Baldeep Singh • \\ Ashish Jaiman • Chandra Shekhar Yadav • \\ Hira Lal Nag
}

Received: 26 July 2008 / Accepted: 28 July 2008 / Published online: 30 August 2008

(C) Springer-Verlag 2008

The treatment of distal third tibial fractures being challenging, we read with great interest the article by Mohammed et al. "Intramedullary tibial nailing in distal third tibial fractures: distal locking screws and fracture non-union" [1].

Modern tibial nail designs have interlocking holes that enable distal placement of screws in close proximity to the tip of the nail. Three distal interlocking holes at 5,15 and $25 \mathrm{~mm}$, respectively, from the nail tip are common in current tibial nails. Also success of at least two interlocking screws in the distal fragment after removal of the distal tip of the traditional nail has been reported [2]. Screw failure and malalignment have been common problems reported in the literature following the use of a single interlocking screw in distal tibial fractures [3, 4]. Distal locking screws have less cortical purchase in metaphyseal bone; as control of the intramedullary nail position in the distal tibial canal depends on these screws, there is increased stress on the screws to maintain fracture alignment [4]. Consequently late complications, in particular loss of reduction, are attributed to implant failure at the distal locking sites of the intramedullary nail [4]. The study [1] however reported that $52 \%$ of the cases had only one distal locking bolt, which is against accepted principles.

Also Mohammed et al. reported that 5 of 12 cases with a single distal screw treated for non-union had unreamed nailing. The unreamed interlocking nail screw system cannot be equated with reamed interlocking nails. Mosheiff

D. S. Neogi $\cdot$ B. Singh $\cdot$ A. Jaiman $\cdot$ C. S. Yadav $\cdot$ H. L. Nag

Department of Orthopaedics,

All India Institute of Medical Sciences,

Ansari Nagar, New Delhi 110029, India

D. S. Neogi $(\bowtie)$

Plot no 2, Michigan Compound,

Saptapur, Dharwad, Karnataka 580001, India

e-mail: drdevdatt@gmail.com et al. [5] reported that 22 of 52 patients with distal tibial metaphyseal fractures treated with unreamed nailing required secondary procedures (e.g. dynamisation, autogenous bone grafting, fibulectomy) to progress to union. Thus the use of unreamed nails may have been an important variable in the non-union cases.

Designed as temporary implants, the unreamed nails can only bear a certain number of load cycles, significantly less than the locking screws of reamed nails. As a consequence, screw failure occurs frequently in unreamed nailing, especially on weight-bearing [6]. In spite of $46 \%$ of the cases in the study by Mohammed et al. having unreamed nailing, they had only one $(0.9 \%)$ incidence of screw breakage. It would be helpful if the authors could detail the postoperative protocol and the method of supplementary protection used, if any, during mobilisation and ambulation in the two subsets of patients as these also influence the screw breakage and healing process.

\section{References}

1. Mohammed A, Saravanan R, Zammit J, King R (2008) Intramedullary tibial nailing in distal third tibial fractures: distal locking screws and fracture non-union. Int Orthop 32:547-549

2. Dogra AS, Ruiz AL, Thompson NS, Nolan PC (2000) Diametaphyseal distal tibial fractures - treatment with a shortened intramedullary nail: a review of 15 cases. Injury 31:799-804

3. Kneifel T, Buckley R (1996) A comparison of one versus two distal locking screws in tibial fractures treated with unreamed tibial nails: a prospective randomized clinical trial. Injury 27(4):271-273

4. Varsalona R, Liu GT (2006) Distal tibial metaphyseal fractures: the role of fibular fixation. Strat Traum Limb Recon 1:42-50

5. Mosheiff R, Safran O, Segal D, Liebergall M (1999) The unreamed tibial nail in the treatment of distal metaphyseal fractures. Injury 30:83-90

6. Boenisch UW, de Boer PG, Journeaux SF (1996) Unreamed intramedullary tibial nailing — fatigue of locking bolts. Injury 27:265-270 